\section{Fatores associados aos biomarcadores inflamatórios em adolescentes: análise por modelagem de equações estruturais}

\author{
Factors associated with inflammatory biomarkers \\ in adolescents: analysis by structural \\ equation modeling
}

\section{Factores asociados a los biomarcadores inflamatorios en adolescentes: análisis por modelado de ecuaciones estructurales}

Eduarda Gomes Bogea 1

Maylla Luanna Barbosa Martins 1

Cadidja Dayane Sousa do Carmo 1

Joelma Ximenes Prado Teixeira Nascimento 1

Soraia Pinheiro Machado Arruda 2

Cecília Claudia Costa Ribeiro 1

Ana Karina Teixeira da Cunha França 1

Antônio Augusto Moura da Silva 1

doi: 10.1590/0102-311X00212220

Correspondência

E. G. Bogea

Universidade Federal do Maranhão.

Rua Barão de Itapari 155, São Luís, MA 65020-070, Brasil.

eduardabogea@gmail.com

1 Universidade Federal do Maranhão, São Luís, Brasil.

2 Universidade Estadual do Ceará, Fortaleza, Brasil. e aluno. O consumo alimentar foi investigado por meio do Questionário de Frequência Alimentar, e os padrões alimentares foram extraídos por análise fatorial por componentes principais. Para avaliar os fatores associados aos biomarcadores inflamatórios, foi utilizada modelagem de equações estruturais. Foram construídas variáveis latentes: condição socioeconômica, que incluiu escolaridade materna, renda familiar e classe econômica; e inflamação, constituída pela interleucina-1B, interleucina-6 e interleucina-8. Um modelo teórico foi elaborado para avaliar fatores associados aos biomarcadores inflamatórios, indicando os efeitos diretos e indiretos entre as variáveis latentes e observadas. Três padrões alimentares foram identificados (ocidental, básico brasileiro e saudável), com variância explicada de $31 \%$, e padrão ocidental o de maior contribuição. Não foram encontradas associações dos padrões com a variável latente inflamação. O excesso de peso foi positivamente associado com a variável latente inflamação (coeficientes padronizados $=0,281 ; p=0,002$ ). $\mathrm{A}$ extração dos três padrões evidencia maior consumo de alimentos ricos em carboidratos e gorduras que são nutrientes diretamente relacionados ao aumento das doenças crônicas não transmissiveis. O excesso de peso foi associado com maiores niveis de biomarcadores inflamatórios.

Inflamação; Ingestão de Alimentos; Estado Nutricional; Adolescente; Análise de Classe Latentes 


\section{Introdução}

Os biomarcadores inflamatórios são proteínas de baixo peso molecular envolvidas no controle metabólico e endócrino do organismo humano. Dosagens elevadas dessas substâncias atuam no mecanismo da inflamação subclínica, envolvida na patogênese de diversas doenças crônicas não transmissíveis (DCNT) 1. Essa resposta inflamatória é mediada e regulada pelas citocinas 2 , que estão associadas aos hábitos alimentares e ao excesso de peso 3 .

No Brasil, essas duas condições - excesso de peso e hábitos alimentares inadequados - têm sido crescentes na adolescência. $\mathrm{O}$ Estudo de Riscos Cardiovasculares em Adolescentes (ERICA), realizado com adolescentes brasileiros de 12 a 17 anos, encontrou prevalências de sobrepeso e obesidade atingindo $17,1 \%$ e $8,4 \%$, respectivamente 4 . Em paralelo, as práticas alimentares nessa fase da vida são caracterizadas por elevado consumo de ultraprocessados e de quantidades insuficientes em frutas e hortaliças, com adesão a dietas monótonas e omissão de refeições ${ }^{5}$. Dessa forma, o conhecimento do consumo alimentar e sua associação com os biomarcadores inflamatórios pode proporcionar um maior entendimento da complexa relação entre dieta e DCNT na adolescência.

A avaliação dos padrões alimentares reflete melhor as condições reais da alimentação 6 e permite fazer associações entre combinações de alimentos, gordura corporal e biomarcadores inflamatórios 3,7. McCourt et al. 7 encontraram associação de padrões alimentares composto por alimentos saudáveis com menor concentração de biomarcadores pró-inflamatórios em adolescentes e adultos jovens.

A atividade física 8 e a situação socioeconômica 9 também podem estar associadas a alterações dos biomarcadores inflamatórios, porém há necessidade de maiores evidências científicas dessas relações em adolescentes. As análises de associação com a modelagem de equações estruturais possibilitam que as relações de variáveis de confusão e mediação sejam avaliadas adequadamente, além de incorporar as variáveis latentes 10 . Dessa forma, o presente estudo tem como objetivo avaliar os fatores associados com os biomarcadores inflamatórios de adolescentes, por meio da variável latente composta pelas interleucina- $1 \beta$, interleucina- 6 e interleucina-8.

\section{Métodos}

\section{Delineamento, população e amostra}

Estudo de delineamento transversal com adolescentes de ambos os sexos, que tinham idade entre 17 e 18 anos, regularmente matriculados em escolas públicas estaduais do Município de São Luís, Maranhão, e realizado no período de janeiro de 2014 a junho de 2016. As informações foram originárias da pesquisa intitulada Os Agravos Bucais em Adolescentes são Marcadores de Risco às Doenças Crônicas não Transmissiveis - ADOLESCER, que obteve aprovação do Comitê de Ética em Pesquisa (CEP) da Universidade Federal do Maranhão (UFMA) sob protocolo no 441.226.

Para a seleção dos alunos, realizou-se amostragem aleatória com conglomeração em três estágios, com sorteio casual simples sem reposição: 1o estágio - escola; 2o estágio - turma e 3o estágio - aluno. No ano de 2013, de acordo com a Secretaria de Estado da Educação do Maranhão (SEDUC), a zona urbana de São Luís, apresentava 50.488 alunos matriculados em 80 escolas de ensino médio. Desses, 42.009 alunos estavam matriculados nas 52 escolas públicas estaduais, tendo sido selecionada de forma randomizada uma amostra de 13 escolas. Posteriormente, foram isolados três níveis de turma de alunos nas escolas previamente selecionadas, gerando 39 classes amostradas. Os estudantes com 17 ou 18 anos de idade $(\mathrm{n}=2.030)$ foram considerados elegíveis para participarem desse estudo. Finalmente, os alunos elegíveis foram randomizados a partir das listas de classe.

Foram incluídos adolescentes matriculados em escolas públicas, de ambos os sexos, na faixa etária entre 17 e 18 anos e que aceitaram participar da pesquisa. Não participaram do estudo aqueles alunos que não frequentavam a escola $(n=62)$, que recusaram participar $(n=109)$, usavam aparelho ortodôntico $(\mathrm{n}=59)$, devido ao maior risco de apresentar doenças periodontais, ou meninas grávidas $(\mathrm{n}=3)$, totalizando 233 alunos não incluídos no estudo.

O tamanho da amostra foi calculado no software EpiInfo, versão 6.0 (https://www.cdc.gov/ epiinfo/index.html). Foram considerados o coeficiente de correlação entre o consumo alimentar e os 
biomarcadores inflamatórios de $15 \%$, o poder do teste de $80 \%$ e o nível de $5 \%$ de significância, sendo estimado uma amostra mínima de 347 adolescentes. Por se tratar de um estudo realizado a partir de dados secundários da referida pesquisa, foram incluídos 391 adolescentes na amostra final.

\section{Procedimento e coleta de dados}

A coleta de dados foi dividida em 3 etapas: na 1a etapa, foram coletadas informações referentes às questões socioeconômicas, demográficas, estilo de vida e história clínica. Nessa ocasião, foi aplicado o Questionário de Frequência Alimentar (QFA), e, aferidos dados antropométricos e pressão arterial. Na 2a etapa, ocorreu a coleta de sangue e a avaliação da composição corporal. Na 3a etapa, realizou-se a entrega dos exames sanguíneos.

As variáveis socioeconômicas, estilo de vida e de história clínica utilizadas foram: sexo (masculino e feminino); renda ( $<1$ salário mínimo, 1 a $<3$ salários mínimos, 3 a $<5$ salários mínimos e $\geq 5$ salários mínimos); classe social proposta pela Associação Brasileira de Empresas de Pesquisa (ABEP. Critério de classificação econômica Brasil. http://www.abep.org/criterio-brasil, acessado em 11/Dez/2018) (A/B, C e D/E), escolaridade da mãe em anos (1-4; 5-8; 9-11; mais de 12); cor da pele (não branca e branca) e atividade física (suficientemente ativo e insuficientemente inativo).

Para a construção da variável latente "situação socioeconômica" (SES), foram utilizadas as seguintes variáveis: (a) escolaridade materna, tratada como variável categórica ordinal; (b) renda familiar foi considerada uma variável categórica, e (c) classe social. As classes A e B formaram a classe alta, mais ricos e escolarizados; C classe média; e D e E foram consideradas como classe baixa, mais pobres e menos escolarizados.

As medidas antropométricas de peso e altura foram aferidas em duplicata. O peso foi obtido em balança digital (Tanita, Santo André, Brasil), e a estatura foi aferida por meio de estadiômetro portátil (Alturexata, Belo Horizonte, Brasil). Para determinação do estado nutricional, foi utilizado o índice de massa corporal (IMC) obtido por meio da razão entre o peso $(\mathrm{kg})$ e a estatura $\left(\mathrm{m}^{2}\right)$, classificado por idade de acordo com a Organização Mundial da Saúde (OMS) para adolescentes 11. Os adolescentes foram classificados em dois grupos: sem excesso de peso e com excesso de peso.

Para avaliação do consumo alimentar, foram utilizados os dados do QFA. O QFA utilizado nesta pesquisa foi adaptado do QFA utilizado no estudo Inquérito de Saúde de São Paulo (ISA-Capital), desenvolvido para a população de 20 anos ou mais, e foi aplicado conforme orientações do manual do ISA-Capital 12. Para obter a variável dietética usada na análise de derivação dos padrões alimentares, a quantidade de alimentos consumidos foi convertida de medidas caseiras para consumo diário de cada alimento em gramas (g) ou mililitros (mL), com o auxílio do manual do ISA-Capital 12.

Os 67 itens alimentares identificados no QFA foram alocados em 21 grupos alimentares, considerando a semelhança da composição centesimal nutricional e o nível de processamento industrial dos alimentos: pães: pão e misto; bolo e biscoito: bolo, biscoito sem recheio e biscoito com recheio; hortaliças = salada, alface, tomate, cenoura, legume, verduras cruas, verduras cozidas, brócolis; frutas = laranja, banana, maçã, melão, mamão, uva e goiaba; suco natural = suco natural; leite e derivados integrais = leite integral, iogurtes de fruta, queijo muçarela; leite e derivados desnatados = leites desnatados, leite semidesnatados, iogurte natural, queijo minas; refrigerante e suco industrializado = refrigerante, refrigerante diet e suco industrializado; café $=$ café sem açúcar e café com açúcar; leguminosas = feijão; cereais $=$ arroz, macarrão, polenta; tubérculos $=$ batata; ovos $=$ ovo; aves e pescados $=$ peixe e frango; carnes $=$ carne de boi e carne de porco; sopa $=$ sopa; farinha $=$ farinha; carnes processadas $=$ carne seca, linguiça, embutidos, nuggets; gorduras = óleo, maionese, margarina e manteiga; temperos = sal e condimentos; bebidas alcoólicas = cerveja e caipirinha; guloseimas = chocolate, achocolatado, sobremesas, sorvetes, gelatinas e bala; ricos em gordura = salgado frito, salgado assado, pizza, batata frita e lasanha; açúcar = açúcar.

Antes da análise do padrões alimentares, foi certificada a adequação do tamanho amostral, pois recomenda-se que o número de indivíduos seja $\geq 5$ para cada alimento/grupo de alimentos do questionário utilizado 13 .

Para avaliação da atividade física, foi utilizado o Questionário de Atividade Física para o Adolescente (QAFA), adaptação do questionário Self-Administered Physical Activity Checklist (SAPAC), validado por Farias-Júnior et al. ${ }^{14}$ para avaliação do nível de atividade física em adolescentes. Para determinar o 
nível de atividade física dos adolescentes, foi realizado o somatório do produto do tempo despendido em cada uma das atividades físicas (minutos/dia) pelas respectivas frequências de prática por semana (dias/semana). Foram considerados suficientemente ativos os adolescentes com prática de atividade física igual ou superior a 300 minutos/semana, e os demais, como insuficientemente ativos.

A coleta de sangue foi realizada nas escolas com os participantes em jejum de 6 horas. As amostras foram mantidas refrigeradas e enviadas para processamento em um laboratório de referência de análises bioquímicas. Os biomarcadores inflamatórios foram determinados pela tecnologia Magpix-Milliplex (https://www.merckmillipore.com/BR/pt/life-science-research/protein-detec tion-quantification/Immunoassay-Platform-Solutions/milliplex-multiplex-assays-using-luminex/ UjGb.qB.8WQAAAE_rn8RHeN.,nav). Para construção da variável latente "Inflamação" (INFLAM), foram utilizados os seguintes biomarcadores inflamatórios, categorizados em tercis: interleucina-1 $\beta$, interleucina-6 e interleucina-8.

\section{Análise estatística}

Foi realizada a caracterização das variáveis socioeconômicas, atividade física e estado nutricional por tercis dos biomarcadores inflamatórios interleucina-1 $\beta$, interleucina- 6 e interleucina-8. Para avaliar a diferença entre os grupos, utilizou-se o teste qui-quadrado por meio do software Stata 14.0 (https:// www.stata.com).

Em relação à variável exposição referente ao consumo alimentar, foram extraídos os padrões alimentares por meio do método Análise de Componentes Principais (ACP), seguido de rotação ortogonal do tipo varimax. A adequação dos dados à análise fatorial foi confirmada por meio do coeficiente de Kaiser-Meyer-Olkin (KMO), sendo considerado adequado o valor de $\mathrm{KMO} \geq 0,60$ 15. O número de fatores retidos foi definido com base nos seguintes critérios: componentes com autovalores maiores que 1,5, gráfico de Cattell (scree plot) e significado conceitual dos padrões identificados. Cada componente principal foi interpretado baseado nos alimentos com cargas fatoriais $\geq 0,30$ ou $\leq-0,30$, que é a carga considerada de importante contribuição para o padrão 16.

Para avaliar os fatores associados aos biomarcadores inflamatórios, foi utilizada modelagem de equações estruturais no software Mplus, versão 8.0 (https://www.statmodel.com/). Foi empregado o método de estimação dos mínimos quadrados (WLSMV) para variáveis observadas categóricas e robusto para não normalidade. A parametrização theta controlou as diferenças de variâncias residuais 17.

Nas análises das estimativas padronizadas para construção das variáveis latentes, considerou-se carga fatorial superior a 0,4 com p < 0,05 como indicativa de que a correlação entre a variável observada e a variável latente é moderadamente alta em magnitude 18.

No modelo teórico (Figura 1), a situação socioeconômica foi considerada um determinante mais distal (variável exógena), exercendo seus efeitos sobre as variáveis dependentes do modelo, como os padrões alimentares, atividade física e excesso de peso. O padrões alimentares e a atividade física teriam efeito sobre excesso de peso corporal e biomarcadores inflamatórios. $\mathrm{O}$ excesso de peso teria efeito sobre os biomarcadores inflamatórios.

Foram considerados valores de índice de ajuste para adequação do modelo: o valor de p maior que 0,05 no teste do qui-quadrado $\left(\chi^{2}\right) ; \mathrm{p}<0,08$ e um limite superior do intervalo de $90 \%$ de confiança (IC90\%) inferior a 0,08 para a raiz do erro quadrático médio da aproximação (RMSEA); índice de ajuste comparativo (CFI) e índice Tucker-Lewis (TLI) $>0,90$ e valor menor que 1 para o índice raiz média ponderada do quadrado residual (WRMR). O qui-quadrado, graus de liberdade e valor de $\mathrm{p}$ foram avaliados, porém não foram adotados como parâmetros para o ajuste do modelo, devido a sua sensibilidade ao tamanho da amostra, e o índice WRMR ainda é considerado experimental, sendo necessário mais testes de suas propriedades 19.

Para obter sugestões de alterações das hipóteses iniciais, o comando modindices foi utilizado. Quando as modificações propostas foram consideradas plausíveis do ponto de vista teórico, um novo modelo foi elaborado e analisado, caso o valor do índice de modificação fosse superior a 10 mil 20. No modelo final, foram avaliados efeitos diretos e indiretos da variável latente e das observadas, considerando-se efeito significativo quando $\mathrm{p}<0,05$. 
Figura 1

Modelo teórico-conceitual de fatores associados aos biomarcadores infamatórios.

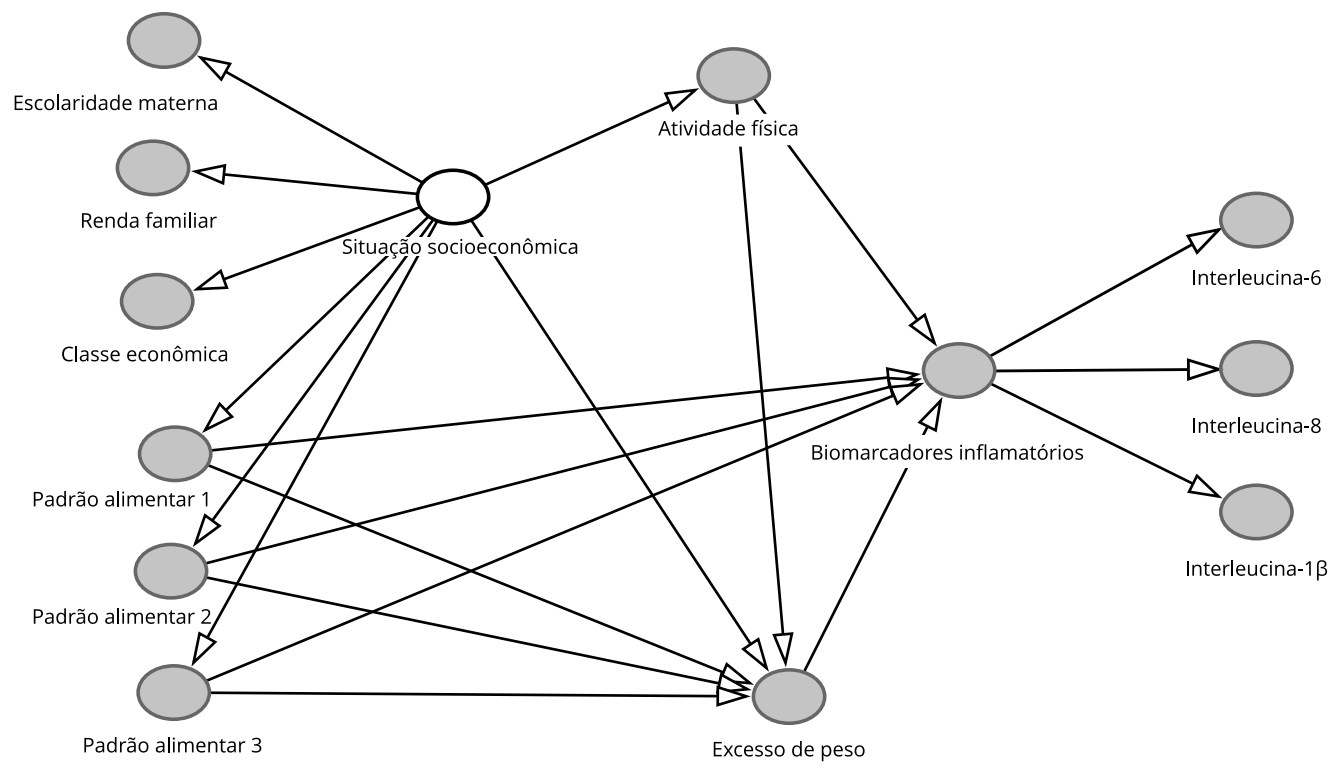

Notas: situação socioeconômica: variável latente da situação socioeconômica familiar do adolescente formada por escolaridade materna, renda e classe econômica (Associação Brasileira de Empresas de Pesquisa. http://www.abep.org/ criterio-brasil, acessado em 11/Dez/2018) da família; padrão alimentar 1: ocidental; padrão alimentar 2: básico brasileiro; padrão alimentar 3: saudável.

\section{Resultados}

Foram avaliados 405 adolescentes, porém, com a exclusão de 3,5\% dos indivíduos pela ausência de aplicação do QFA, a amostra do presente estudo foi composta por 391 adolescentes, sendo 56,3\% do sexo feminino. Observou-se que 47,3\% tinham renda familiar de 1 a 3 salários mínimos, 40,2\% das suas mães tinham entre 9 e 11 anos de estudos, e 84,4\% eram de cor da pele não branca, e 64,4\% estavam inseridas na classe socioeconômica C. Em relação a avaliação do estado nutricional, 16,9\% dos adolescentes tinham excesso de peso, e 50,6\% eram suficientemente ativos.

Os tercis dos biomarcadores interleucina- $1 \beta$, interleucina-6, interleucina- 8 variaram entre: $1,09 \mathrm{pg} / \mathrm{mL}-10,31 \mathrm{pg} / \mathrm{mL} ; 1,74 \mathrm{pg} / \mathrm{mL}-84,39 \mathrm{pg} / \mathrm{mL}$ e $46,01 \mathrm{pg} / \mathrm{mL}-894,43 \mathrm{pg} / \mathrm{mL}$, respectivamente. Percebeu-se uma maior prevalência dos adolescentes com excesso de peso no maior tercil da interleucina-6 $(\mathrm{p}=0,003)$ e de adolescentes da cor branca no maior tercil da interleucina- $8(\mathrm{p}=0,028)$ (Tabela 1).

Para identificação dos padrões alimentares, o valor do teste para avaliação das correlações entre os itens alimentares e da adequação do uso da análise fatorial foi satisfatório ( $\mathrm{KMO}=0,741)$. Considerando-se o scree plot (4 pontos no maior declive), autovalores $\geq 1,5$ e interpretações dos padrões extraídos, o número de componentes retido foi no total de três padrões, explicando 31\% da variância total. Os padrões alimentares foram definidos da seguinte forma: ocidental (guloseimas, junk food, sopas, bolo/biscoito com recheio, refrigerante/suco industrializado, carnes processadas, carnes vermelhas), composto por alimentos ultraprocessados e carne vermelha; básico brasileiro (óleos e gorduras, açúcar de adição, pães/biscoito sem recheio, café, farinha, cereais, leites e derivados integrais, ovos, leguminosas), composto por alimentos típicos da alimentação do brasileiro; saudável (suco natural, 
Tabela 1

Características socioeconômicas, atividade física e estado nutricional por tercis dos biomarcadores interleucina-1 $\beta$, interleucina- 6 e interleucina-8 em adolescentes da pesquisa Os Agravos Bucais em Adolescentes são Marcadores de Risco às Doenças Crônicas não Transmissíveis - ADOLESCER, São Luís, Maranhão, Brasil, 2014-2016.

\begin{tabular}{|c|c|c|c|c|c|c|c|c|c|c|c|c|}
\hline & \multicolumn{4}{|c|}{ Interleucina-1 $\beta$} & \multicolumn{4}{|c|}{ Interleucina-6 } & \multicolumn{4}{|c|}{ Interleucina-8 } \\
\hline & T1 (\%) & T2 (\%) & T3 (\%) & Valor de p & T1 (\%) & T2 (\%) & T3 (\%) & Valor de $p$ & T1 (\%) & T2 (\%) & T3 (\%) & Valor de $p$ \\
\hline Sexo & & & & 0,395 & & & & 0,150 & & & & 0,949 \\
\hline Masculino & 34,19 & 44,52 & 21,29 & & 38,71 & 32,90 & 28,39 & & 33,60 & 32,80 & 33,60 & \\
\hline Feminino & 32,99 & 39,59 & 27,41 & & 28,93 & 37,06 & 34,01 & & 32,68 & 34,64 & 32,68 & \\
\hline Classe econômica & & & & 0,055 & & & & 0,217 & & & & 0,052 \\
\hline A/B & 43,48 & 37,68 & 18,84 & & 30,43 & 30,43 & 39,13 & & 44,68 & 14,89 & 40,43 & \\
\hline C & 33,19 & 39,66 & 27,16 & & 35,78 & 34,05 & 30,17 & & 30,32 & 38,30 & 31,38 & \\
\hline $\mathrm{D} / \mathrm{E}$ & 21,56 & 56,86 & 21,57 & & 25,49 & 47,06 & 27,45 & & 32,56 & 34,88 & 32,56 & \\
\hline Cor da pele & & & & 0,107 & & & & 0,849 & & & & 0,028 * \\
\hline Não branca & 32,78 & 40,47 & 26,76 & & 33,11 & 34,78 & 32,11 & & 34,02 & 35,66 & 30,33 & \\
\hline Branca & 37,74 & 49,06 & 13,21 & & 33,96 & 37,74 & 28,30 & & 26,47 & 20,59 & 52,94 & \\
\hline Atividade física & & & & 0,112 & & & & 0,381 & & & & 0,499 \\
\hline Suficientemente ativo & 32,58 & 46,63 & 20,79 & & 30,90 & 34,23 & 34,83 & & 35,82 & 30,60 & 33,58 & \\
\hline Insuficientemente ativo & 34,48 & 36,78 & 38,74 & & 35,63 & 36,21 & 28,16 & & 30,56 & 36,81 & 32,64 & \\
\hline Estado nutricional & & & & 0,950 & & & & 0,003 * & & & & 0,328 \\
\hline Sem excesso de peso & 33,56 & 42,03 & 24,41 & & 36,27 & 35,59 & 28,14 & & 33,48 & 35,19 & 31,33 & \\
\hline Com excesso de peso & 33,33 & 40,35 & 24,72 & & 17,54 & 33,33 & 49,12 & & 31,11 & 26,67 & 42,22 & \\
\hline
\end{tabular}

* $p<0,05$

frutas, aves e pescados, tempero, tubérculos, hortaliças e leite desnatado e derivados), constituído por alimentos in natura e minimamente processados. O padrão ocidental explicou a maior proporção da variância total $(12,4 \%)$.

O modelo teórico inicial, incluindo as variáveis alergia e aleitamento materno exclusivo, não teve bom ajuste segundo os índices CFI e TLI. O segundo modelo teórico analisado em modelagem de equações estruturais com a exclusão das variáveis alergia e aleitamento materno mostrou um bom ajuste para todos os índices considerados $(\mathrm{RMSEA}=0,023$, CFI $=0,962$, TLI $=0,938$ e WRMR $=$ 0,667) (Tabela 2) e foi construído pelas variáveis latentes: SES e INFLAM, prática de atividade física, estado nutricional e pressão arterial. As variáveis latentes SES e INFLAM mostraram cargas fatoriais para os indicadores $>0,40$ e valor de p significativos, indicando adequada validade convergente (Tabela 3).

O excesso de peso teve efeito total e direto positivos nos maiores níveis séricos dos biomarcadores inflamatórios, com o coeficiente padronizado - CF de 0,281 (p=0,002), tanto para o efeito total como para o efeito direto. Não foi encontrada associação significativa nas demais variáveis do modelo (situação socieconômica, atividade física e padrões alimentares) (Tabela 4). 
Tabela 2

Índices de ajuste para modelagem de equações estruturais para a associação entre os padrões alimentares e a inflamação subclínica em adolescentes em São Luís, Maranhão, Brasil, 2014-2016.

\begin{tabular}{|c|c|c|}
\hline Índices & Índices esperados & Modelo teórico final \\
\hline Teste $\chi^{2}$ & & 41175 \\
\hline Graus de liberdade & & 34 \\
\hline Valor de $p$ do teste $\chi^{2}$ & & 0,1854 \\
\hline Raiz do erro quadrático médio da aproximação (RMSEA) & $<0,05$ & 0,023 \\
\hline Intervalo de $90 \%$ de confiança & $<0,08$ & $0,000-0,046$ \\
\hline Valor de $\mathrm{p}$ & & 0,980 \\
\hline Índice de ajuste comparativo (CFI) & $>0,90$ & 0,962 \\
\hline Índice Tucker-Lewis & $>0,90$ & 0,938 \\
\hline Raiz média ponderada do quadrado residual (WRMR) & $<1,00$ & 0,667 \\
\hline
\end{tabular}

Tabela 3

Cargas fatoriais, erros padrões e valores de p para indicadores das variáveis latentes.

\begin{tabular}{|c|c|c|c|}
\hline \multirow[t]{2}{*}{ Variável latente } & \multicolumn{3}{|c|}{ Modelo teórico } \\
\hline & Carga fatorial & Erro padrão & Valor de p \\
\hline \multicolumn{4}{|l|}{ Situação socioeconômica * } \\
\hline Escolaridade materna & 0,572 & 0,085 & $<0,001$ \\
\hline Renda familiar & 0,455 & 0,098 & $<0,001$ \\
\hline $\mathrm{ABEP} * \star$ & 0,748 & 0,115 & $<0,001$ \\
\hline \multicolumn{4}{|l|}{ Inflamação *** } \\
\hline Interleucina-1 $\beta$ (tercil) & 0,439 & 0,066 & $<0,001$ \\
\hline Interleucina-6 (tercil) & 0,734 & 0,080 & $<0,001$ \\
\hline Interleucina-8 (tercil) & 0,685 & 0,089 & $<0,001$ \\
\hline
\end{tabular}

* Situação socioeconômica (SES): variável latente da situação socioeconômica familiar do adolescente formada por escolaridade materna e renda familiar;

** ABEP: Associação Brasileira de Empresas de Pesquisa (Critério de classificação econômica Brasil. http://www.abep. org/criterio-brasil, acessado em 11/Dez/2018)

*** Inflamação (INFLAM): variável latente formada pela interleucina-1 $\beta$, interleucina-6 e interleucina-8, em tercis.

\section{Tabela 4}

Efeitos total e direto na associação entre a variável latente inflamação com os padrões alimentares, estado nutricional e atividade física.

\begin{tabular}{|c|c|c|c|c|c|c|}
\hline \multirow[t]{3}{*}{ Variável } & \multicolumn{6}{|c|}{ Modelo teórico } \\
\hline & \multicolumn{3}{|c|}{ Efeito total da inflamação * } & \multicolumn{3}{|c|}{ Efeito direto da inflamação * } \\
\hline & $\begin{array}{l}\text { Coeficiente } \\
\text { padronizado }\end{array}$ & Erro padrão & Valor de p & $\begin{array}{c}\text { Coeficiente } \\
\text { padronizado }\end{array}$ & Erro padrão & Valor de p \\
\hline Situação socioeconômica & 0,056 & 0,088 & 0,527 & 0,050 & 0,092 & 0,582 \\
\hline Padrão alimentar ocidental & 0,009 & 0,078 & 0,905 & 0,005 & 0,076 & 0,950 \\
\hline Padrão alimentar básico brasileiro & 0,049 & 0,071 & 0,488 & 0,040 & 0,072 & 0,578 \\
\hline Padrão alimentar saudável & $-0,046$ & 0,072 & 0,526 & $-0,051$ & 0,076 & 0,502 \\
\hline Excesso de peso & 0,281 & 0,093 & 0,002 & 0,281 & 0,093 & 0,002 \\
\hline Prática de atividade física & 0,023 & 0,088 & 0,791 & 0,036 & 0,089 & 0,682 \\
\hline
\end{tabular}

* Inflamação (INFLAM): variável latente formada pela interleucina-1 $\beta$, interleucina-6 e interleucina-8, em tercis. 


\section{Discussão}

Três padrões alimentares foram identificados: ocidental, básico brasileiro e saudável, sendo o padrão ocidental com maior contribuição na variância proporcional. A variável latente inflamação foi positivamente associada ao excesso de peso.

Como limitações do estudo, destaca-se o tipo de desenho transversal, estando sujeito a vieses, como o da causalidade reversa. Além disso, o efeito da dieta e atividade física sobre a ocorrência de desfechos de caráter crônico tem maior possibilidade de ser evidenciado em estudos com desenho longitudinal. Por ter sido desenvolvido apenas com adolescentes de escolas da rede pública de ensino, os resultados não podem ser generalizados para os adolescentes de escolas privadas. $\mathrm{O}$ instrumento de avaliação de consumo alimentar utilizado também apresenta limitações relacionadas à memória, inerente aos inquéritos retrospectivos, e ao fato de ter sido adaptado do QFA desenvolvido para uma população adulta de uma capital do Sudeste do país. Apesar disso, o QFA é um dos métodos mais indicados para estimar o consumo alimentar habitual dos indivíduos por ser capaz de capturar a probabilidade de consumo da maioria dos alimentos.

Destacam-se, como pontos fortes do estudo, a utilização de biomarcadores inflamatórios em uma fase precoce da vida e os métodos estatísticos utilizados, como a modelagem de equações estruturais para avaliar associação e a extração de padrões alimentares por meio de ACP.

No presente estudo, o padrão "ocidental" explicou o maior percentual da variância proporcional, melhor representando o consumo alimentar da amostra avaliada. Esse padrão alimentar é formado por alimentos ultraprocessados e carne vermelha, sendo ricos em açúcares refinados, gorduras saturadas, aditivos químicos e sódio. Souza et al. 21, no ERICA realizado em 124 cidades brasileiras com adolescentes de 12 a 17 anos, também observaram elevada prevalência de consumo de alimentos como refrigerantes, salgados fritos e assados, biscoitos doces e salgados. A redução do consumo desses alimentos é uma das recomendações do novo Guia Alimentar para População Brasileira, pois um elevado consumo aumenta o risco de obesidade e DCNT 7,21. A nomenclatura desse padrões alimentares considerado não saudável nos estudos com adolescentes varia em ocidental 3,22, junk food 5,16, fast food 23, obesogênico 24 e não saudável 25 .

O segundo padrão alimentar, denotado de "básico brasileiro", é constituído por alimentos comumente consumidos pela população brasileira diariamente e de fácil acesso, fazendo parte da cultura alimentar brasileira (arroz e feijão) e maranhense (farinha). Do ponto de vista nutricional, esse padrão possui predominantemente alimentos ricos em carboidratos, simples e complexos. O estudo ERICA 21 encontrou elevadas taxas de consumo para o arroz (82\%) e o feijão (68\%) nos adolescentes estudados, apontando uma forte influência cultural nas escolhas alimentares. Salvatti et al. 16 e Rodrigues et al. 22 encontraram padrão bem similar nos seus estudos.

O padrão "alimentar saudável" foi composto por alimentos in natura e minimamente processados, com um maior aporte de vitaminas, minerais, fibras, proteína, além de baixo teor de açúcares simples, ácidos graxos saturados e trans. Os alimentos constituídos nesse padrão são considerados protetores para a saúde cardiovascular, importantes no aumento da capacidade de respostas imunológicas e na prevenção de aparecimento de DCNT 7 . Dentre os padrões extraídos, esse apresentou a menor variância proporcional, o que aponta para um menor consumo pelos adolescentes dos alimentos desse padrão. Estudo no Nordeste brasileiro, com 3.392 adolescentes entre 14 e 19 anos, observou que $88,6 \%$ não cumpriam as recomendações de consumo de frutas, legumes e verduras 26 , concordando com o menor consumo desses grupos alimentares neste estudo.

As diferenças entre os padrões em composição com a mesma denominação encontrados na literatura podem ser explicadas pela subjetividade inerente aos métodos de ACP. Para este estudo, a maioria dos componentes encontrados reflete a percepção dos principais grupos de alimentos da adolescência, coincidindo com as práticas mais comuns de alimentação nessa fase, com maior consumo de alimentos ricos em carboidratos refinados e gordura saturada e menor de frutas e hortaliças 25 .

Nenhum dos padrões alimentares encontrados associou-se ao estado nutricional dos indivíduos avaliados. Esse resultado é similar ao de Bibilone et al. ${ }^{3}$ em estudo na Espanha, que não encontraram associação de sobrepeso e obesidade com os padrões ocidental e mediterrâneo em adolescentes do sexo feminino. Pinho et al. 24 encontraram que os adolescentes brasileiros apresentavam uma maior 
prevalência de sobrepeso/obesidade no segundo tercil do padrão "obesogênico" em comparação com o terceiro tercil, com a direção da associação contrária ao esperado.

Esse resultado pode ser explicado por causalidade reversa, uma limitação de estudos transversais. Os adolescentes, percebendo que estavam acima do peso, poderiam reduzir a ingestão de alimentos do padrão ocidental, que são conhecidos por ser potencialmente obesogênicos, e aumentar o consumo de alimentos do padrão saudável. Outro aspecto relevante é a baixa prevalência de excesso de peso da amostra, com valores similares as mais baixas prevalências da América Latina, apontada por Rivera et al. 27 em uma revisão sistemática.

Os padrões alimentares não foram associados à variável latente inflamação. Tal achado pode ser justificado pelo tempo das consequências das escolhas alimentares sobre a ocorrência de desfechos sistêmicos, muitas vezes, ainda, não sendo evidenciadas em populações jovens como essa. Além disso, o tamanho amostral pode não ter sido suficiente para a detecção da associação entre o padrões alimentares e um efeito de pequeno, bem como homogeneidade da amostra, por ser constituída de alunos somente de escola pública. Estudos em adolescentes saudáveis são escassos, principalmente que associem padrões ou hábitos alimentares com biomarcadores de inflamação, resultando em poucas informações disponíveis sobre os efeitos dos padrões alimentares na inflamação.

Diferentemente aos achados do presente estudo, outros autores encontraram associação entre os padrões alimentares e biomarcadores inflamatórios. Estudo realizado no nordeste da Irlanda com adolescentes e adultos jovens identificou que concentrações de homocisteína foram menores nos indivíduos que tiveram uma maior adesão aos padrões "saudável" e "social" e maiores para o padrão "ocidental" 7. Bibiloni et al. 3, ao investigarem adolescentes do sexo feminino na Espanha, encontraram uma maior aderência ao padrão "ocidental" associada com menor concentração plasmática de adiponectina e maior de homocisteína e interleucina-6. Em relação ao padrão "Mediterrâneo", foi encontrado maior concentração plasmática de adiponectina.

Apesar da ausência de associação do padrões alimentares com a variável latente inflamação, o presente estudo identificou que o excesso de peso teve efeitos total e direto positivos nessa variável, chamando a atenção para uma alteração sistêmica desencadeada pelo excesso de peso corporal já numa fase precoce da vida. Tal achado pode ser explicado por meio da teoria que, em indivíduo com excesso de peso, os adipócitos ficam hipertrofiados, ocasionando uma compressão da vasculatura do tecido e reduzindo a chegada de oxigênio. Em consequência à hipóxia, haveria um estímulo à produção de citocinas pró-inflamatórias a fim de aumentar a angiogênese e o fluxo sanguíneo ${ }^{2}$. Os resultados encontrados são consistentes com estudos prévios, que apontam o aumento da inflamação subclínica em adolescentes com excesso de peso 28.

Em virtude da associação encontrada, pode-se afirmar que a inflamação subclínica é detectável por meio do IMC em adolescentes. Bibioni et al. 3 observou que as medidas do IMC foram preditoras de níveis plasmáticos de três biomarcadores inflamatórios: adiponectina, leptina e PCR-ultrassensível. Esses dados apontam para a importância da utilização desse indicador, que, além de simples, fácil, de baixo custo para classificar o estado nutricional, também prediz sobre a inflamação subclínica.

A extração dos três padrões evidencia o maior consumo de alimentos ricos em amido, gorduras e açúcares, nutrientes diretamente relacionados com o aumento das DCNT. Maior concentração dos biomarcadores foi associada ao estado nutricional avaliado a partir do IMC, podendo esse indicador ser considerado um importante preditor de inflamação subclínica em adolescentes.

Esses resultados apontam para a necessidade de ações de conscientização para a melhoria das práticas alimentares, por meio de políticas públicas de incentivo ao maior consumo de alimentos saudáveis e redução de alimentos ultraprocessados e ricos em carboidratos simples. Além disso, chama a atenção para a contenção da epidemia da obesidade, por estar diretamente relacionada à inflamação subclínica, aumentando o risco de DCNT. 


\section{Colaboradores}

E. G. Bogea e A. K. T. C. França contribuíram na concepção e projeto, análise e interpretação dos dados, redação do artigo e revisão crítica relevante do conteúdo intelectual. M. L. B. Martins contribuiu na redação do artigo e revisão crítica relevante do conteúdo intelectual. C. D. S. Carmo e J. X. P. T. Nascimento contribuíram na concepção e no projeto, análise e interpretação dos dados, e aprovação final da versão a ser publicada. S. P. M. Arruda e A. A. M. Silva contribuíram na análise, e interpretação dos dados. C. C. C. Ribeiro contribuiu na concepção e no projeto de estudo. Todos os autores revisaram e aprovaram a versão final a ser publicada do texto.

\section{Informações adicionais}

ORCID: Eduarda Gomes Bogea (0000-0003-26568238); Maylla Luanna Barbosa Martins (00000002-6402-3899); Cadidja Dayane Sousa do Carmo (0000-0002-5139-2441); Joelma Ximenes Prado Teixeira Nascimento (0000-0002-2871-8685); Soraia Pinheiro Machado Arruda (0000-00023918-4738); Cecília Claudia Costa Ribeiro (00000003-0041-7618); Ana Karina Teixeira da Cunha França (0000-0002-4460-2631); Antônio Augusto Moura da Silva (0000-0003-4968-5138).

\section{Agradecimentos}

Os autores são gratos à Fundação de Amparo à Pesquisa do Estado do Maranhão (FAPEMA), ao Conselho de Desenvolvimento Científico e Tecnológico (CNPq) e ao Departamento de Ciência e Tecnologia (DECIT), Ministério da Saúde pelo apoio financeiro.

\section{Referências}

1. Castro-Piñero J, Ortega FB, Ruiz JR. Adiposity and cardiovascular risk in children and adolescents: implications of the amount of fat carried and where. Mayo Clin Proc 2019; 94:1928-30.

2. Saltiel AR, Olefsky JM. Inflammatory mechanisms linking obesity and metabolic disease. J Clin Invest 2017; 127:1-4.

3. Bibiloni MM, Maffeis C, Llompart I, Pons A, Tur JA. Dietary factors associated with subclinical inflammation among girls. Eur J Clin Nutr 2013; 67:1264-70.

4. Bloch KV, Klein CH, Szklo M, Kuschnir MCC, Abreu GDA, Barufaldi LA, et al. ERICA: prevalências de hipertensão arterial e obesidade em adolescentes brasileiros. Rev Saúde Pública 2016; 50 Suppl 1:9s

5. Pinho L, Fernandes FE, Sousa SSH, Botelho ACDC, Caldeira AP. Excesso de peso e consumo alimentar em adolescentes de escolas públicas no norte de Minas Gerais, Brasil. Ciênc Saúde Colet 2014; 19:67-74.

6. Carvalho CA, Fonsêca PC, Nobre LN, Priore SE, Franceschini SDCC. Metodologias de identificação de padrões alimentares a posteriori em crianças brasileiras: revisão sistemática. Ciênc Saúde Colet 2016; 21:143-54.

7. McCourt HJ, Draffin CR, Woodside JV, Cardwell CR, Young IS, Hunter SJ, et al. Dietary patterns and cardiovascular risk factors in adolescents and young adults: the Northern Ireland Young Hearts Project. Br J Nutr 2014; 112:1685-98.

8. Alizadeh H, Safarzade A, Talebi-Garakani E, Khandan M, Arab Z, Saadat F, et al. Impact of high-intensity interval training and circuit resistance training protocols on serum levels of interleukin-10, lipid profile, body composition and insulin resistance index in overweight male adolescents. Jentashapir Journal of Cellular and Molecular Biology 2019; 10:e79324.

9. Carmeli C, Steen J, Petrovic D, Lepage B, Delpierre C, Kelly-Irving M, et al. Mechanisms of life-course socioeconomic inequalities in adult systemic inflammation: findings from two cohort studies. Soc Sci Med 2020; 245:112685.

10. Silva AAM, Vasconcelos AGG, Bettiol H, Barbieri MA. Socioeconomic status, birth weight, maternal smoking during pregnancy and adiposity in early adult life: an analysis using structural equation modeling. Cad Saúde Pública 2010; 26:15-29.

11. World Health Organization. Who child growth standards: lenght/height-for-age, weightfor-lenght, weight-for-height and body mass index-for-age. Methods and development. Geneva: World Health Organization; 2007.

12. Fisberg RM, Lobo DM. Manual de avaliação do consumo alimentar em estudos populacionais: a experiência do inquérito de saúde em São Paulo (ISA). http://www.gac-usp.com.br/ resources/manual\%20isa\%20biblioteca\%20 usp.pdf (acessado em 20/Mar/2019). 
13. Pestana MH, Gageiro JN. Análise de dados para ciências sociais: a complementaridade do SPSS. Lisboa: Edições Sílabo; 2005.

14. Farias Júnior JC, Lopes AS, Mota J. Validade e reprodutibilidade de um questionário para medida de atividade física em adolescentes. Rev Bras Epidemiol 2012; 15:198-210.

15. Newby PK, Tucker KL. Empirically derived eating patterns using factor or cluster analysis: a review. Nutr Rev 2004; 62:177-203.

16. Salvatti AG, Escrivão MAMS, Taddei JAAC, Bracco MM. Eating patterns of eutrophic and overweight adolescents in the city of São Paulo, Brazil. Rev Nutr 2011; 24:703-13.

17. Muthen LK, Muthen BO. Mplus: statistical analysis with latent variables. User's guide (1998-2010). 6th. Ed. Los Angeles: Muthén \& Muthén; 2010.

18. Kline RB. Principles and practice of structural equation modeling. New York: The Guilford Press; 2011.

19. Byrne BM. Structural equation modeling with Mplus: basic concepts, applications and programming. New York: Routledge; 2012.

20. Wang J, Wang X. Structural equation modeling: applications using Mplus. Noida: Thomson Digital; 2012.

21. Souza ADM, Barufaldi LA, Abreu GDA, Giannini DT, Oliveira CLD, Santos MMD, et al. ERICA: ingestão de macro e micronutrientes em adolescentes brasileiros. Rev Saúde Pública 2016; 50 Suppl 1:5s

22. Rodrigues PRM, Pereira RA, Cunha DB, Sichieri R, Ferreira MG, Vilela AAF, et al. Fatores associados a padrões alimentares em adolescentes: um estudo de base escolar em Cuiabá, Mato Grosso. Rev Bras Epidemiol 2012; 15:662-74.
23. Dishchekenian VR, Escrivão MA, Palma D, Ancona-Lopez F, Araújo EACD, Taddei JAAC. Padrões alimentares de adolescentes obesos e diferentes repercussões metabólicas. Rev Nutr 2011; 24:17-29.

24. Pinho MGMD, Adami F, Benedet J, Vasconcelos FDAGD. Association between screen time and dietary patterns and overweight/obesity among adolescents. Rev Nutr 2017; 30:377-89.

25. Tavares LF, Castro IRR, Levy RB, Cardoso LDO, Claro RM. Dietary patterns of Brazilian adolescents: results of the Brazilian National School-Based Health Survey (PeNSE. Cad Saúde Pública 2014; 30:2679-90.

26. Silva FMA, Smith-Menezes A, Silva Duarte MDF. Consumo de frutas e vegetais associado a outros comportamentos de risco em adolescentes no Nordeste do Brasil. Rev Paul Pediatr 2016; 34:309-15.

27. Rivera JA, Cossío TG, Pedraza LS, Aburto TC, Sánchez TG, Martorell R. Childhood and adolescent overweight and obesity in Latin America: a systematic review. Lancet Diabetes Endocrinol 2014; 2:321-32.

28. Caprio S, Perry R, Kursawe R. Adolescent obesity and insulin resistance: roles of ectopic fat accumulation and adipose innflammation. Gastroenterology 2017; 152:1638-46. 


\section{Abstract}

The study aimed to analyze factors associated with inflammatory biomarkers in adolescents. This was a cross-sectional study in 391 adolescents 17-18 years of age in public schools in São Luís, Maranhão State, Brazil. Three-stage random cluster sampling was performed in schools, classes, and students. Food consumption was studied with a food frequency questionnaire, and dietary patterns were extracted by principal components factor analysis. Assessment of factors associated with inflammatory biomarkers used structural equation modeling. Latent variables were constructed: socioeconomic status, which included maternal schooling, family income, and economic class; and inflammation, consisting of interleukin- $1 \beta$, interleukin-6, and interleukin-8. A theoretical model was developed to assess factors associated with inflammatory biomarkers, indicating direct and indirect effects between the latent and observed variables. Three dietary patterns were identified (Western, basic Brazilian, and healthy), with $31 \%$ explained variance and the Western pattern with the largest share. No associations were found between the patterns and the latent variable inflammation (standardized coefficients $=0.281$; $p=0.002$ ). Extraction of the three patterns evidenced higher consumption of foods high in carbohydrates and fats, which are nutrients associated directly with the increase in chronic noncommunicable diseases. Excess weight was associated with higher levels of inflammatory biomarkers.

Inflammation; Eating; Nutritional Status; Adolescent; Latent Class Analysis

\section{Resumen}

El objetivo fue evaluar los factores asociados con biomarcadores inflamatorios en adolescentes. Se trata de un estudio transversal con 391 adolescentes entre 17 e 18 años de escuelas públicas en São Luís, Maranhão, Brasil. Se realizó una muestra aleatoria por conglomerados en tres etapas: escuela, grupo y alumno. El consumo alimentario fue investigado mediante el cuestionario de frecuencia alimentaria, $y$ los patrones alimentarios fueron extraídos por análisis factorial por componentes principales. Para evaluar los factores asociados a los biomarcadores inflamatorios se utilizó un modelado de ecuaciones estructurales. Se construyeron variables latentes: condición socioeconómica, que incluyó escolaridad materna, renta familiar y clase económica; e inflamación, constituida por la interleucina-1 $1 \beta$, interleucina- 6 e interleucina- 8 . Un modelo teórico se elaboró para evaluar factores asociados a los biomarcadores inflamatorios, indicando los efectos directos e indirectos entre las variables latentes y observadas. Se identificaron tres patrones alimentarios (occidental, básico brasileño y saludable), con variancia explicada de un $31 \%$ y patrón occidental o de mayor contribución. No se encontraron asociaciones de los patrones con la variable latente inflamación. El exceso de peso estuvo positivamente asociado con la variable latente inflamación (coeficientes estandarizados $=0,281 ; p=0,002)$. La extracción de los tres patrones evidencia mayor consumo de alimentos ricos en carbohidratos y grasas que son nutrientes directamente relacionadas con el aumento de las enfermedades crónicas no transmisibles. El exceso de peso estuvo asociado con mayores niveles de biomarcadores inflamatorios.

Inflamación; Ingestión de Alimentos; Estado Nutricional; Adolescente; Análisis de Clases Latentes
Recebido em 21/Jul/2021

Versão final reapresentada em 07/Fev/2021 Aprovado em 26/Fev/2021 